\title{
A Case of Tumor-Induced Osteomalacia: Finding the Culprit Acetabular Tumor and Successful Resection with a Novel Hip Joint- Preserving Surgery
}

\author{
Christie G. Turin ${ }^{1}$, Robert J. Wilson' ${ }^{2}$ Ciera Mangone ${ }^{3}$, Karen Rosenspire ${ }^{4}$ Jessica Berman ${ }^{1}$, \\ Michelle Walker ${ }^{1}$, Ravi Amaravadi, Mona Al Mukaddam ${ }^{1}$
}

\section{Learning Point of the Article:}

This innovative hip joint-sparing technique could provide surgical cure to patients with TIO of the acetabulum without performing total hip arthroplasty.

\section{Abstract}

Introduction: Tumor-induced osteomalacia (TIO) is a rare paraneoplastic disorder caused by tumors that produce fibroblast growth factor 23 (FGF23) resulting in phosphate wasting and inadequate bone mineralization. Complete resection of the tumor can be curative. However, these tumors are typically difficult to find anatomically due to small size and location.

Case Report: We present the case of a patient who presented for evaluation of recurrent fractures and hypophosphatemia in the setting of elevated FGF23 suggestive of TIO. 68Gallium-DOTATATE revealed multiple somatostatin avid lesions in several ribs, left acetabulum, sacrum, right tibia, and feet, some of which appeared with fracture on computed tomography scan, initially concerning for metastatic disease. However, the lesion in acetabulum was considered the culprit tumor given its remarkably higher maximum standard uptake values. Complete surgical removal of the FGF23-secreting tumorled to cure of this disease.

Conclusion: This case report highlights the challenges with functional imaging differentiating fractures from the culprit lesion and reports on a novel surgical technique that allowed for surgical cure while preserving the hip joint.

Keywords: Hypophosphatemia, osteomalacia, tumor, functional imaging, surgical technique.

\section{Introduction}

Tumor-induced osteomalacia (TIO), also known as oncogenic osteomalacia, is a rare paraneoplastic disorder caused by tumors that produce fibroblast growth factor 23 (FGF23) resulting in hypophosphatemia and inadequate bone mineralization leading to muscle weakness, bony pain and recurrent fractures [1].

Given the rarity of this condition and because phosphorus levels are not checked on routine labs, the diagnosis of TIO is often delayed for several years. Once the diagnosis is suspected, the next crucial step is to identify the culprit lesion, given that complete surgical resection is curative. The localization of these mesenchymal tumors is challenging because these lesions are typically small and can be found anywhere in the body. Although whole body functional imaging is considered the firstline diagnostic modality, there is a high rate of false positive. Given the location of those lesions and the need for en bloc resection with wide margins, surgery could introduce significant morbidity. This case highlights the challenges with functional imaging and reports on a novel surgical technique that allowed for surgical cure while preserving the hip joint.

Author's Photo Gallery
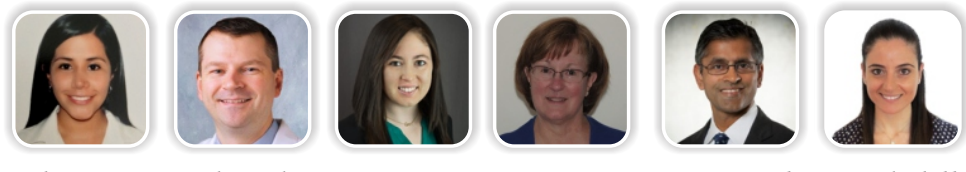

Dr. Ravi Amaravadi Dr. Mona Al Mukaddam

Department of Medicine, Division of Endocrinology, Diabetes and Metabolism, The University of Pennsylvania, Philadelphia, PA, United States. ${ }^{2}$ Department of Orthopaedic, Hospital of the University of Pennsylvania, Philadelphia, PA, United States,

${ }^{3}$ Department of Pathology, Hospital of the University of Pennsylvania, Philadelphia, PA, United States,

"Department of Radiology, Hospital of the University of Pennsylvania, Philadelphia, PA, United States,

${ }^{5}$ Department of Hematology and Oncology, Hospital of the University of Pennsylvania, Philadelphia, PA, United States.

10.13107/jocr.2021.v11.i03.2078

Address of Correspondence

Dr. Christie G Turin,

Endocrinology Fellow at the Hospital of the University of Pennsylvania, 3400 Civic Center Blvd., Philadelphia, PA 19104, United States.

E-mail: christie.turinmore@pennmedicine.upenn.edu

Journal of Orthopaedic Case Reports | pISSN 2250-0685 | eISSN 2321-3817 | Available on www.jocr.co.in | doi:10.13107/jocr.2021.v11.i03.2078 This is an Open Access article distributed under the terms of the Creative Commons Attribution Non-Commercial License (http://creativecommons.org/licenses/by-nc/3.0) which permits unrestricted non-commercial use, distribution, and reproduction in any medium, provided the original work is properly cited. 


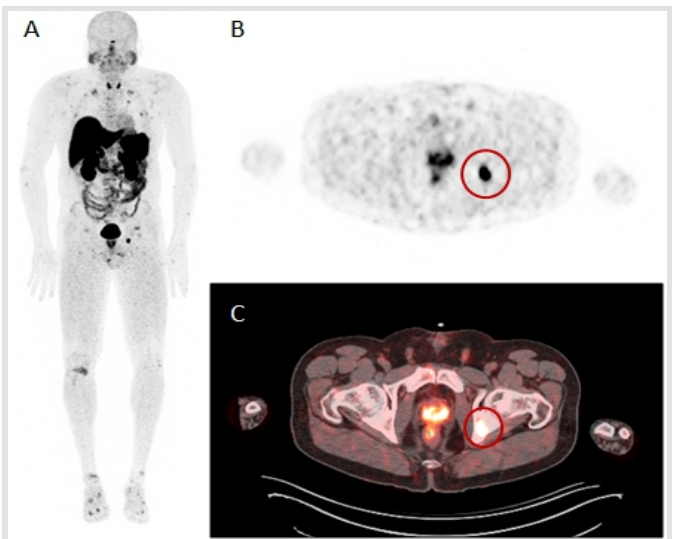

Figure 1: 68 Gallium-DOTATATE positron emission tomography/computed tomography (PET/CT). Abnormal uptake in multiple fractures of ribs, right tibia, ankles, and feet (a). PET and CT scan showed abnormal uptake (SUVmax 20.4) in left posterior acetabulum in red circle ( $b$ and $c)$.

\section{Case Report}

A 43-year-old man with primary hypothyroidism presented for evaluation of progressive fatigue, weakness, muscle and joint pain in setting of recurrent fractures.

He was first diagnosed with non-traumatic left ankle fracture at age 41, which was treated conservatively. A few weeks after this diagnosis, he began to experience musculoskeletal pain in several locations including bilateral knees, ankles, left shoulder, left arm, and lower back which affected his overall functional status. He struggled to climb a flight of stairs, often grabbing the railing to pull himself up. A nuclear medicine bone scan showed abnormal uptake in bilateral ribs, vertebrae, right knee, hips, ankles, and feet. A lower extremity MRI showed stress fractures in left fibula, fifth metatarsal, and right tibia.

The patient was first seen at our Bone Center in March 2019. Laboratory evaluation showed normal calcium at $9.3 \mathrm{mg} / \mathrm{dL}$, low phosphorus at $1.5 \mathrm{mg} / \mathrm{dL}$, low 1,25-dihydroxyvitamin $\mathrm{D}$ at $13 \mathrm{pg} / \mathrm{mL}$, high phosphate excretion fraction in urine (27\%), elevated alkaline phosphatase at $163 \mathrm{U} / \mathrm{L}$, and elevated FGF23 at $238 \mathrm{RU} / \mathrm{mL}$ (Table 1). The patient started calcitriol and phosphate supplements that significantly improved muscle weakness; however, he continued to have bony pain.

Due to concern for TIO, a positron emission tomography/computed tomography (PET/CT) scan with 68Gallium $(68 \mathrm{Ga})$-DOTATATE was performed which showed multiple lytic osseous lesions with increased somatostatin positive uptake seen throughout pelvis, ribs, ankles, and feet concerning for metastatic disease (Fig. 1). However, after rereview with radiology, multiple areas of increased uptake were considered to be secondary to fractures, and left posterior acetabular lesion was presumed to be the tumor of interest given a remarkably higher standard uptake values (SUVmax) in this lesion compared to other lesions (SUVmax20 vs.4-5).

Although it is not recommended to perform biopsy of presumed lesion due to risk for tumor cell seeding [3], the left acetabular lesion was biopsied given concern for metastatic disease. The biopsy results showed: Spindle cell neoplasm present. The patient underwent radical resection of the tumor of the posterior left acetabulum with reconstruction of the posterior wall and posterior column of the acetabulum through a Kocher-Langenbach approach. His hip joint was able to be spared utilizing a 3D-printed custom bone cutting guide (CG) and intra-operative $\mathrm{O}$-arm guided surgical navigation (Medtronic@ Stealth) utilizing a navigated Sonopet Ultrasonic Bone Cutting Bovie (Stryker) (Fig. 2). The posterior wall and column of his acetabulum were constructed with a tantalum augment (Zimmer) and a pelvic reconstruction plate and screw construct (Synthes) after tumor removal.

Pathology demonstrated a $1.4 \mathrm{~cm}$ phosphaturic mesenchymal tumor of the left acetabulum with uninvolved margins (Fig. 3). FGF23 normalized within $24 \mathrm{~h}$ after surgery $(127 \mathrm{RU} / \mathrm{mL})$ and calcitriol and phosphate supplements were discontinued on post-operative day 10. Phosphorus and FGF23 levels remained normal at $4.8 \mathrm{mg} / \mathrm{dL}$ and $83 \mathrm{RU} / \mathrm{mL}$, respectively, 6 weeks after surgery.
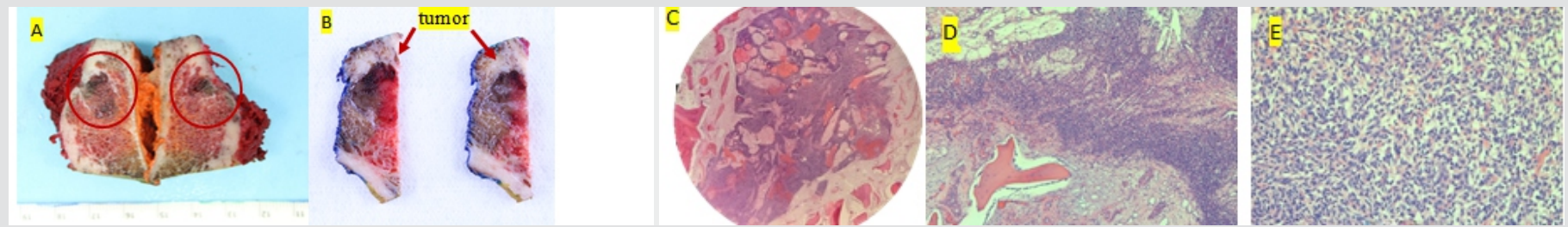

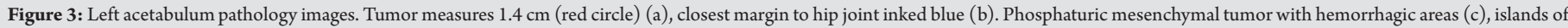
entrapped trabecularbone (d), and sheets of bland spindle cells (e) H and E, original magnification $\times 2.5(\mathrm{c}), \times 20$ (d), $\times 40$ (e) 


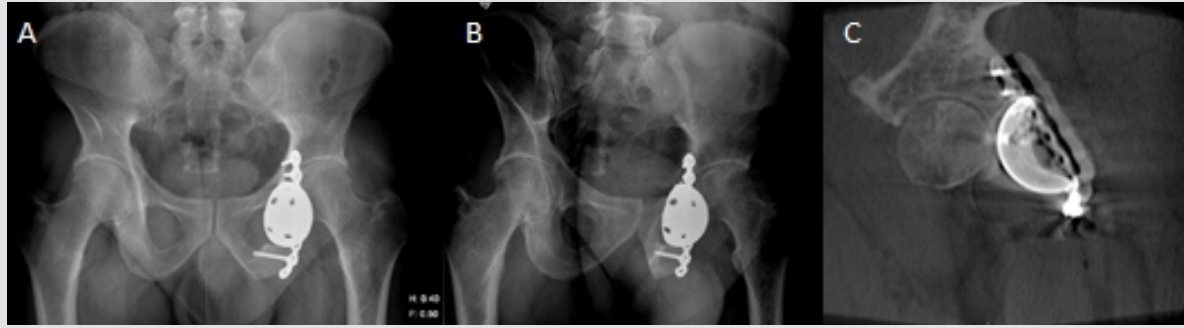

Figure 4: Post-operative imaging. ( $\mathrm{a}$ and $\mathrm{b}$ ) X-rays of reconstruction showing tantalum augment filling the void and stabilized with additional posterior column plate. (c) Sagittal post-operative computed tomography scan showing hip joint-sparing reconstruction.

Postoperatively, he was kept toe-touch weight bearing for 6 weeks and then advanced to weight bearing as tolerated over the subsequent 6 weeks. Posterior hip precautions were employed for 3 months postoperatively. Clinical and radiographic followup at 6 months postoperatively found him to be pain-free in his left hip, with no evidence of hardware complication (Fig. 4), back to hiking, and walking on the treadmill. The patient has continued to report improvement in his symptoms, functional status has returned to baseline, with almost complete resolution of bone pain, and no recurrence of fractures. Phosphorus level also remained normal at $3.4 \mathrm{mg} / \mathrm{dL} 6$ months after surgery.

\section{Discussion}

Phosphorus is a critical element in skeletal development, bone mineralization, cellular signaling, cellular membrane composition, among other functions. It is regulated by the kidneys, bones, intestines, and parathyroid glands. FGF23, a protein produced by the bone, is one of the regulators of phosphate hemostasis [2].

TIO is a very rare paraneoplastic syndrome characterized by phosphate wasting [1]. It is most commonly caused by phosphaturic mesenchymal tumors, osteoblastoma-like tumors, ossifying fibrous-like tumors, and non-ossifying fibrous-like tumors [1]. Of relevance, these tumors do not have a clear anatomical predilection but are recognized by their ability to produce FGF23. FGF23 decreases the expression of sodium-phosphate IIa/IIc co-transporters in renal brush border membrane which leads to increased phosphate renal excretion. FGF23 also affects Vitamin D metabolism by decreasing circulating levels of $1 \alpha$ hydroxylase and increases (from 2 months to 19 years) after symptoms start to establish the diagnosis $[3,4]$. Biochemical findings, in addition to low phosphorus and low 1,25-dihydroxyvitamin $\mathrm{D}$ as mentioned above, are elevated alkaline phosphatase, elevated FGF23 levels, and elevated fractional excretion of urinary phosphate.

One of the biggest challenges is to detect the culprit lesion due to small size and variable location. There are three different modalities of tests that are used to localize the lesion: Functional imaging, anatomical imaging, and venous sampling. Functional imaging, specifically 68Ga-DOTATATE PET/CT, is currently considered the first step $[3,5]$. Phosphaturic mesenchymal tumors express somatostatin receptors, mainly the subtype $2 \mathrm{~A}$, which can be identified using radio-labeled somatostatin analogs such as $68 \mathrm{Ga}$.

Retrospective [6] and prospective [7] studies have evaluated the efficacy of 68Ga-DOTATATE PET/CT to localize the culprit lesions. Zhang et al. [6] reviewed the PET/CT images and clinical charts of 54 patients with suspected TIO. They found that 44 patients had a positive scan, among which 32 confirmed diagnoses by surgical resection. One patient was found to have granuloma instead of TIO, and 11 patients did not undergo surgery; therefore, they were not included in the analysis as the diagnosis could not be confirmed by pathology. They propose a sensitivity of $100 \%$ and a specificity of $90 \%$. On the other hand, El-Maouche et al. [7] conducted a prospective study comparing functional studies in 11 patients with TIO based on biochemical findings. Patients underwent $68 \mathrm{Ga}$ DOTATATE PET/CT in addition to octreoscan-SPECT/CT and 18 F FDG-PET/CT. TIO was identified in $6 / 11$ cases by $68 \mathrm{Ga}$ DOTATATE, resulting in a sensitivity of $55 \%$ and

\section{Table 1: Laboratory evaluation. Since time of initial visit and during follow-up.}

\begin{tabular}{|c|c|c|c|c|c|c|c|c|}
\hline Date & $\begin{array}{l}\text { Calcium mg/dL } \\
(8.9-10.3)\end{array}$ & $\begin{array}{c}\text { Albumin g/dL } \\
(3.6-5.1)\end{array}$ & $\begin{array}{c}\text { Phosphorus mg/dL } \\
(2.4-4.7)\end{array}$ & $\begin{array}{l}\text { ALP IU/L } \\
(38-126)\end{array}$ & $\begin{array}{l}\text { iPTH pg/mL } \\
(1.6-6.9)\end{array}$ & $\begin{array}{c}25-\mathrm{OH} \text { D ng/mL } \\
(25-80)\end{array}$ & $\begin{array}{c}1,25 \mathrm{OH}_{2} \mathrm{D} \mathrm{pg} / \mathrm{mL} \\
(19.9-79.3)\end{array}$ & $\begin{array}{c}\text { FGF } 23 \mathrm{RU} / \mathrm{ml} \\
(<180)\end{array}$ \\
\hline Initial visit & 9.3 & 4.5 & 1.5 & 163 & 5.7 & 36 & 13 & 268 \\
\hline POD\#3 & 8.4 & 3.1 & 2.7 & 107 & & & & \\
\hline POD\#7 & 8.8 & 3.6 & 2.5 & & & & & \\
\hline POD\#14 & & & 4.6 & & 5.9 & & 54 & \\
\hline
\end{tabular}

Journal of Orthopaedic Case Reports | Volume 11 | Issue 3 | March 2021 | Page 37-41 
specificity of $86 \%$. Both octreoscan-SPECT/CT and $18 \mathrm{~F}$ FDG-PET/CT identified the tumor in 4/11 cases.

Although 68Ga-DOTATATE PET/CT is a sensitive and specific technique, other physiologic and pathologic processes could also demonstrate high uptake of the radiotracer due to increased osteoblastic activity. SUVmax values have been directly correlated with expression of somatostatin receptor subtype $2[8]$. Osteoblastic processes such as fractures, degenerative bone disease, vertebral hemangiomas involve high expression of somatostatin receptor subtype 2, which causes increased uptake in PET/CT scan [9] and higher SUVmax. In our patient's case, he had multiple fractures that showed increased uptake; however, degree of uptake differed significantly from culprit lesion when compared by SUVmax.

After identification of culprit lesion, complete surgical resection can lead to cure. Sun et al. [10] reported outcomes of 40 patients with TIO in extremities who underwent surgical resection. Two of 40 patients had bone tumors in femoral head and underwent total hip replacement achieving cure. Three of 40 patients had bone tumors in greater trochanter and underwent tumor curettage due to difficult location; however, one of them required repeat surgical intervention and prosthesis reconstruction afterward. The authors concluded that compared with tumor curettage surgery, surgical resection seemed a better option for management of TIO.

TIO located in the acetabulum has been previously reported. Nakamura et al. [11] reported a case of phosphaturic mesenchymal tumor of the acetabulum that required total hip arthroplasty using a Muller support ring. Given location, patients are usually treated with total hip arthroplasty. Our patient did not undergo total hip replacement, but instead had complete resection of tumor with reconstruction of posterior wall of acetabulum (Fig. 2). We are aware of no other cases of TIO lesions being cured by surgical removal while sparing a major joint with 3D-printed CGs or surgical navigation. Such technology is applied typically to bone sarcomas. In addition, the tantalum augment is used in a novel way as they are typically reserved for revision hip replacement surgeries, not hip sparing surgeries. This case, to the best of our knowledge, is the first report that describes complete surgical resection using a hip joint-preserving technique with surgical navigation for management of TIO.

\section{Conclusion}

Early recognition of culprit lesion in TIO is crucial, as successful surgery is curative and would lead to significant improvement in the quality of life of patients. Functional imaging with $68 \mathrm{Ga}-$ DOTATATE PET/CT is recommended for localization of the culprit tumor. In the setting of multiple areas of uptake comparing SUVmax and correlation with CT scan findings is important to differentiate between fractures and tumors. In addition, this novel surgical technique performed through a Kocher-Langenbeck approach utilizing a 3D-printed custom bone CG provides the advantage of achieving surgical cure while preserving the hip joint.

\section{Clinical Message}

Comparing SUVmax and reviewing anatomical imaging is a fundamental step to localize the culprit lesion in TIO. In regards to treatment options, innovative surgical techniques with custom $3 \mathrm{D}$-printed bone CG could provide surgical cure without performing total hip arthroplasty.

\section{References}

1. Jan de Beur SM. Tumor-induced osteomalacia. JAMA 2005;294:1260-7.

2. Vervloet M. Renal and extrarenal effects of fibroblast growth factor 23. Nat Rev Nephrol2019;15:109-20.

3. Minisola S, Peacock M, Fukumoto S. Tumour-induced osteomalacia. Nat Rev Dis Primers 2017;3:1-5.

4. Drezner MK. Tumor-induced osteomalacia. Rev Endocr Metab Disord 2001;2:175-86.

5. Rayamajhi SJ, Yeh R, Wong T, Dumeer S, Mittal BR, Remotti $\mathrm{F}$, et al. Tumor-induced osteomalacia-current imaging modalities and a systemic approach for tumor location. Clin Imaging 2019;56:114-23.

6. Zhang J, Zhu Z, Zhong D, Dang Y, Xing H, Du Y, et al. 68Ga DOTATATE is an accurate imaging modality in the detection of culprit tumors causing osteomalacia. Clin Nucl Med 2015;40:642-6.

7. El-Maouche D, Sadowski SM, Papadakis GZ, Guthrie L, Cottle-Delisle C, Merkel R, et al. 68Ga DOTATATE for tumor localization in tumor-induced osteomalacia. J Clin Endocrinol Metab 2016;101:3575-81.

8. Boy C, Heusner TA, Poeppel TD, Redmann-Bischofs A, Unger N, Jentzen W, et al. 68Ga-DOTATOC PET/CT and somatostatin receptor (sst1-sst5) expression in normal human tissue: Correlation of sst 2 mRNA and SUVmax. Eur J Nucl Med Mol Imaging 2011;38:1224-36.

9. Hofman MS, Lau WF, Hicks RJ. Somatostatin receptor imaging with $68 \mathrm{Ga}$ DOTATATE PET/CT: Clinical utility, normal patterns, pearls, and pitfalls in interpretation. 
Radiographics 2015;35:500-16.

10. Sun ZJ, Jin J, Qiu GX. Surgical treatment of tumor-induced osteomalacia: A retrospective review of 40 cases with extremity tumors. BMC Musculoskelet Disord $2015 ; 16: 43$.

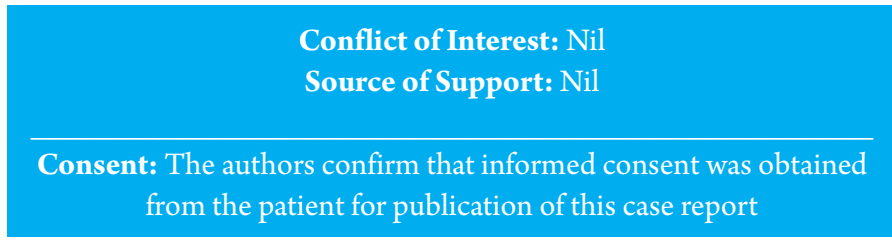

11. Nakamura K, Ohishi M, Matsunobu T, Nakashima Y, Sakamoto A, Maekawa A, et al. Tumor-induced osteomalacia caused by a massive phosphaturic mesenchymal tumor of the acetabulum: A case report. Mod Rheumatol2018;28:906-10.

\section{How to Cite this Article}

Turin CG, Wilson RJ, Mangone C, Rosenspire K, Berman J, Walker M, Amaravadi R, Al Mukaddam M. A Case of Tumor-Induced Osteomalacia: Finding the Culprit Acetabular Tumor and Successful Resection with a Novel Hip Joint-Preserving Surgery. Journal of Orthopaedic Case Reports 2021 March;11(3):37-41. 